\title{
CHROMOSPHERIC AND CORONAL SPECTRA
}

\author{
CAROLE JORDAN \\ Department of Physics (Theoretical Physics), University of \\ Oxford, 1, Keble Road, Oxford, OX1 3NP, UK
}

The interpretation of chromospheric and coronal spectra requires accurate ionization and recombination rates, collision strengths and transition probabilities. Recent projects to improve calculations of opacities in stellar interiors have led to a large amount of new atomic data. Some current and potential applications of atomic data to chromospheric and coronal spectra are mentioned below.

Strong chromospheric lines are optically thick, and the solution of the radiative transfer equations can depend on atomic data for other species contributing to the background opacity. Many lines in the spectra of stars with hot coronae are excited by electron collisions, but in the cooler non-coronal giants radiative processes involving the $H$ Lyman $\alpha$ and $\beta$ lines become more important (see Jordan 1988a). Photo-ionization rates from ground configuration excited terms and oscillator strengths to high levels are still needed.

Fe II is an important ion producing emission lines in stellar chromospheres. Several excitation mechanisms contribute to the observed spectra (Jordan 1988b). Permitted lines to the ground term and low lying metastable terms have high optical depths and transfer photons to spin forbidden lines sharing a common upper level (e.g. mults. uv 1 and uv 3 transfer photons to mults. uv 32 and 61). Line intensity ratios yield the optical depth in the optically thick lines. The strong H Ly $\alpha$ line in cool giants and supergiants excites high levels in Fe II, resulting in strong decays in multiplets such as uv 391 and 399. A large number of f-values are required to interpret the lines formed by these radiative processes. Nahar \& Pradhan (1994) have published some results from the Opacity Project, calculated by using the close coupling method and observed energy levels (which introduce some allowance for relativistic effects). In most cases these $\mathrm{f}$-values agree with experimental results and the calculations by Kurucz (1988) to within 10\%. The latter are still needed for the interpretation of stellar uv spectra because of the treatment of spin-forbidden lines.

Excitation by ion-electron collisions and optical continuum radiation are important in late-type giant stars (Judge et al. 1992). Comparisons of emission measures of effectively optically thin lines, for three giant stars and the Sun, show that the $z^{6} \mathrm{D}^{0}$ and ${ }^{6} \mathrm{~F}^{0}$ terms are excited by collisions, but the $\mathrm{z}^{6} \mathrm{P}^{0},{ }^{4} \mathrm{P}^{0},{ }^{4} \mathrm{D}^{0}$ and ${ }^{4} \mathrm{~F}^{0}$ terms are excited from metastable levels by photospheric radiation. However, collisions dominate in main-sequence stars, where Fe II is 
a major contributor to the total chromospheric radiation losses (Anderson \& Athay 1989). Pradhan \& Berrington (1993) have published some collision strengths, but further data are required, including those of the most important spin-forbidden transitions.

There have been concerted efforts to improve the collision strengths required for the interpretation of solar coronal line fluxes. The existing data have been reviewed in preparation for the SOHO mission (see Lang 1994). Regarding ion populations, in particular of iron, there are differences between those used in the current (August 1994) versions of popular power loss codes, used in analyzing stellar EUV and X-ray data (those by Raymond \& Smith (RS), see Raymond 1988; Mewe \& Kaastra (MK), see Mewe et al. 1985, Kaastra 1992, Landini \& Monsignori Fossi (LMF) 1990). These arise primarily from the treatment of di-electronic recombination. The RS code uses the general formula of Burgess (1965) with corrections suggested by Smith et al. (1985) for additional autoionization routes, but the MK code uses the ion balance of Arnaud \& Rothenflug (ARo) (1985) in which the rates are from Jacobs et al. (1977). For ions above about Fe XV these are significantly smaller because of the larger correction made for the above process. Thus the MK code gives lower temperatures for the peak ion fractions than the RS code for Fe XVI to XXI, but for higher ions the results are very similar. For Fe IX to XIV the RS ion fractions peak at similar or lower temperatures than do those of ARo. There are some systematic, but not large differences between the peak ion fractions. Arnaud \& Raymond (ARa) (1992) bave reviewed the ionization and recombination rates for the Fe ions. For $\mathrm{Fe} X$ to Fe XVII the new results do not differ significantly from those used previously in the RS code, the main difference is the lower peak temperature found for Fe IX. In Fe XVIII to XXIV, the new peak temperatures are higher than in either ARo or the RS code calculations, because of the higher dielectronic recombination rates used by ARa. For Fe XVII to XXVI the new results of ARa agree closely with those of Jordan (1970), because essentially the same rates have been used. For most ions the peak ion populations and temperatures found by LMF lie between those of ARo and those of ARa or of Jordan $(1969,1970)$. The main future requirement is for sets of ion populations as a function of $\mathrm{N}_{e}$.

Regarding the number of transitions treated, of the "current" codes the MK code includes the largest number of lines below $296 \AA$, and the LMF code includes more individual transition than the RS code. Some types of transitions are still not included in the codes, or are treated only approximately, for example, transitions of the type $\Delta \mathrm{n}=1, \Delta \mathrm{l}=$ 0,2 in Fe IX to Fe XIV, and $\Delta \mathrm{n}=1, \Delta \mathrm{l}=0$ in Fe XVIII to XXII. However, more accurate rates are available in the literature from the work of Sampson and his colleagues. The decays from the above levels in Fe IX to Fe XIV will contribute to the background of weak lines in the EUVE short wavelength region.

Anderson, L.S. \& Athay, R.G. 1989, ApJ, 346, 1010

Arnaud, M. \& Rothenflug, R. 1985, A\&AS, 60, 425

Arnaud, M. \& Raymond, J.C. 1992, ApJ, 389, 394

Burgess, A. 1965, ApJ, 141, 1588

Jacobs, V.L., Davis, J., Kepple, P.c. \& Blaha, M. 1977, ApJ, 211, 605 
Jordan, C. 1969 , MNRAS, 142, 499

Jordan, C. 1970, MNRAS, 148, 17

Jordan, C. 1988a, J. Opt. Soc. Am. B, Vol. 5, No. 10, 2252

Jordan, C. 1988b, in Viotti, R., Vittone, A. \& Friedjung, M., eds, Physics of Formation of Fe II Lines outside LTE, p.223

Judge, P.G., Jordan, C. \& Feldman, U. 1992, ApJ, 384, 613

Kaastra, J.E. 1992, Internal SRON-Leiden Report, V2

Kurucz, R.L. 1988, Private Communication, results available on magnetic tape.

Landini, M. \& Monsignori Fossi, B.C. 1990, A\&AS, 82, 229

Lang, J. 1994, Special ed, Atomic Data Nucl. Data, Vol. 57

Mewe, R., Gronenschild, E.H.B.M. \& van den Oord, G.H.J. 1985, A\&AS, 62, 197

\title{
INTERPRETING THE SPECTRA OF CHEMICALLY PECULIAR STARS
}

\author{
THIERRY LANZ
}

\section{Universities Space Research Association NASA/GSFC, Greenbelt MD, USA}

Similarly to many astrophysical studies, a spectroscopic analysis of chemically peculiar (CP) stars requires extensive, accurate sets of atomic data. Our purpose is to show here that the CP stars can also serve as a laboratory for checking the accuracy of the atomic data. Despite additional challenges toward modeling the atmospheres of the CP stars, they have a definite advantage as laboratories for atomic physics: their strong chemical anomalies make some spectral features especially prominent in their spectra, which can be better checked against theoretical predictions. This statement may be applied to both to stars exhibiting large over- or under- abundances. We discuss several examples how CP stars can be used along this idea.

We will focus here on the chemically peculiar stars of the upper main-sequence, which encompass the classical Am and Ap stars. Chemical anomalies are widespread on the main sequence for spectral types B to F. These stars mostly show weak belium lines, and abnormally strong lines from iron-peak and heavier (e.g. the lanthanides) elements. A classical characteristics of Am stars in their weak $\mathrm{Ca}$ lines, while Ap stars may exhibit either strong $\mathrm{Si}$ or $\mathrm{Hg}$ lines. CP stars can be grouped according to the most obvious line strength anomalies, which are related to the effective temperature and to the presence or the absence of a magnetic field. In magnetic $C P$ stars, the chemical elements are not uniformly distributed over the stellar surface, but they seem to be concentrated (or depleted) in rings or polar caps following the magnetic geometry. Magnetic CP stars have also broad absorption features in their ultraviolet and visible spectra that can used photometric peculiarity criteria. Using the new extensive photoionization data from the Opacity Project (OP), the UV absorption features can be identified and the UV spectrum can be well 